\title{
Toward an Improved Wind Inversion Algorithm for RapidScat
}

\author{
Wenming Lin, Marcos Portabella, Ad Stoffelen, and Anton Verhoef
}

\begin{abstract}
The sea-surface winds from the RapidScat scatterometer (RSCAT) onboard the International Space Station (ISS) have been produced using the Pencil-beam scatterometer Wind Processor (PenWP) since December 2014. An inversion residual or Maximum Likelihood Estimator (MLE-) based quality control (QC) algorithm is included in PenWP to distinguish between good- and poor-quality winds. Generally, the QC-accepted RSCAT winds are in good agreement with both the Advanced Scatterometer (ASCAT) winds and the European Centre for Medium-range Weather Forecasting (ECMWF) model output. In contrast, the QC-rejected winds present an overall positive bias with respect to ASCAT and ECMWF winds, mainly due to the impact of rain. However, it has been recently found that a considerable portion $(>5 \%)$ of RSCAT QC-rejected contains anomalously low retrieved speeds $(w<4 \mathrm{~m} / \mathrm{s})$ under medium or high wind conditions $(w>10 \mathrm{~m} / \mathrm{s})$ according to ASCAT/ECMWF. This paper attempts to sort out the cause for these spuriously low winds. A revised MLE inversion with prefiltering the anomalous backscatters is proposed to correct the mentioned inversion issue. The impact of such improved inversion on the retrieved RSCAT winds is evaluated using both the collocated ASCAT and ECMWF winds. The results show that the proposed algorithm improves the wind retrieval of the spuriously low wind cases remarkably, while preserving about $4.7 \%$ of the nominal QC-rejected data $(0.25 \%$ in total).
\end{abstract}

Index Terms-Anomalous backscatter, low winds, quality control, rapidScat scatterometer, wind inversion.

\section{INTRODUCTION}

$\mathbf{T}$ HE RapidScat scatterometer (RSCAT) was installed on the International Space Station (ISS) on September 21, 2014, and has been producing sea-surface wind data since the beginning of October 2014. RSCAT is a conically scanning pencil-beam radar system operating at $\mathrm{Ku}$-band $(13.4 \mathrm{GHz})$ frequency, much like the SeaWinds instrument onboard QuikSCAT satellite [1]. In fact, RSCAT was primarily constructed from

Manuscript received June 24, 2016; revised September 9, 2016; accepted October 7, 2016. Date of publication December 6, 2016; date of current version May 24, 2017. This work was supported in part by the European Organization for the Exploitation of Meteorological Satellites Numerical Weather Prediction Satellite Application Facility Associated Scientist project under Reference NWP-AS16-2 and in part by the Spanish Ministry of Economy and Competitiveness (MINECO) R\&D project under Reference ESP2015-67549-C3-2-R (Corresponding author: Wenming Lin.)

W. Lin and M. Portabella are with the Department of Physical Oceanography, Institute of Marine Sciences, 08003 Barcelona, Spain (e-mail: wenminglin@ icm.csic.es; portabella@icm.csic.es).

A. Stoffelen and A. Verhoef are with the Department of Weather Research, Royal Netherlands Meteorological Institute, 3730 AE De Bilt, The Netherlands (e-mail: ad.stoffelen@knmi.nl; verhoefa@knmi.nl).

Color versions of one or more of the figures in this paper are available online at http://ieeexplore.ieee.org.

Digital Object Identifier 10.1109/JSTARS.2016.2616889
QuikSCAT's spare parts. Consequently, the ocean surface winds can straightforwardly be retrieved from RSCAT backscatter (normalized radar cross section, $\sigma^{\circ}$ ) measurements using the SeaWinds wind processor. The Royal Netherlands Meteorological Institute (KNMI) has adapted the European Organization for the Exploitation of Meteorological Satellites (EUMETSAT) Numerical Weather Prediction Satellite Application Facility (NWP SAF) SeaWinds Data Processor (SDP) to a more general NWP SAF Pencil-beam scatterometer Wind Processor (PenWP) [2], [3]. Since December 2014, the RSCAT winds have been produced by PenWP at KNMI, based on the Level 2A backscatter data, kindly provided by the National Aeronautics and Space Administration's Jet Propulsion Laboratory (JPL), and disseminated through the EUMETSAT Ocean and Sea Ice (OSI) SAF [4].

PenWP includes a traditional Maximum Likelihood Estimator (MLE-) based quality control (QC) algorithm to sort the good-quality winds from the poor-quality winds [3], [5]. That is, large inconsistencies between the different backscatter data from a single wind vector cell (WVC) and the wind Geophysical Model Function (GMF) lead to a large inversion residual or MLE value. A set of MLE thresholds, which are a function of wind speed and across track position or WVC number, are then used to discriminate the good-quality wind retrievals (with lower MLE value) from the poor-quality retrievals (with higher MLE value). When compared to the European Centre for Medium-range Weather Forecasting (ECMWF) real $10 \mathrm{~m}$ winds, the RSCAT wind speed bias is $0.11 \mathrm{~m} / \mathrm{s}$ for both the $25-\mathrm{km}$ and the $50-\mathrm{km}$ QC-accepted data. The standard deviations of the wind zonal $(u)$ and the meridional $(v)$ wind components are below $1.3 \mathrm{~m} / \mathrm{s}$ for the $25-\mathrm{km}$ product and $1.2 \mathrm{~m} / \mathrm{s}$ for the $50-\mathrm{km}$ product [4]. However, the RSCAT QC algorithm has not been fully assessed yet. Since the PenWP QC algorithm is adapted from SDP, one may expect that the distribution of RSCAT "poor-quality" winds is similar to that of the SeaWinds rejected data. That is, for low and moderate wind conditions, the QC-rejected data have in general a large positive bias w.r.t. ECMWF winds due to the impact of rain at $\mathrm{Ku}$-band [4]. At such wind regimes, the latter is dominated by the rain volume backscatter, which increases the total backscatter power thus leading to spuriously retrieved high winds. However, it has been recently found that there are about $5 \%$ of RSCAT QC-rejected data with spuriously low wind speed (retrieved $w<4 \mathrm{~m} / \mathrm{s}$ ) under medium or high wind conditions (reference wind $w>10 \mathrm{~m} / \mathrm{s}$ ), as shown in Fig. 1. This has never been reported in the OSI SAF Pencil-beam scatterometers wind products. 


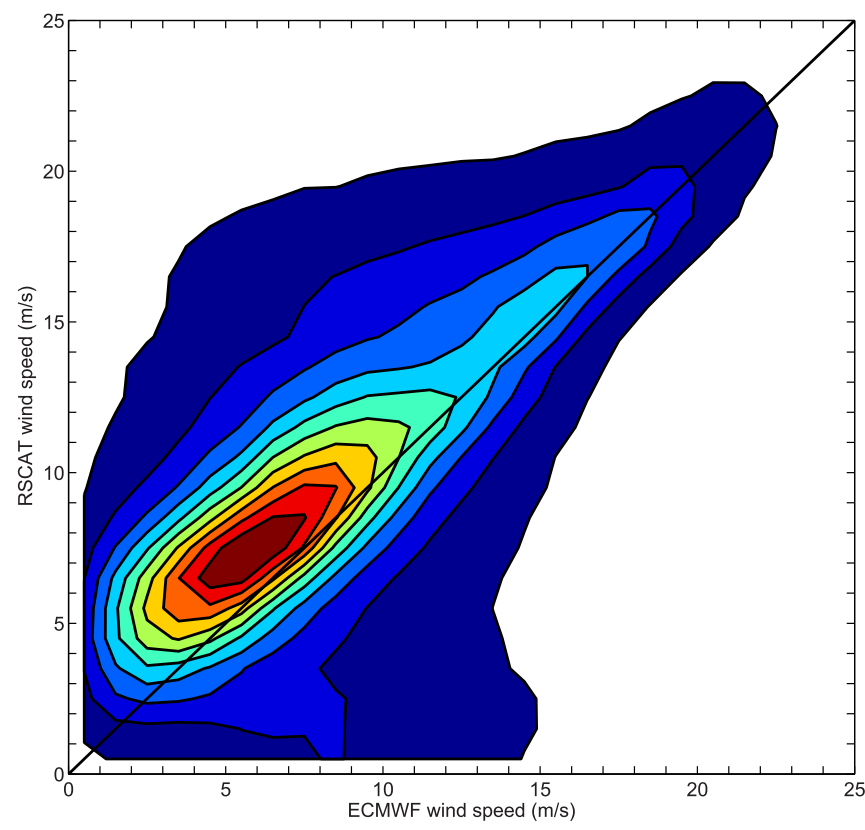

Fig. 1. Two-dimensional histogram of RSCAT QC-rejected data versus ECMWF winds (bins of $1 \mathrm{~m} / \mathrm{s}$ ) in January 2015. The lowest contour line level is $2 \%$, and the following steps are $10 \%, 20 \%, .$. , and $90 \%$ of the maximum data bin.

This paper presents a revised wind inversion algorithm that aims to improve the quality of the PenWP-derived RSCAT winds, in particular of the spuriously low wind speeds. Section II provides an overview of the PenWP wind inversion algorithm, in particular the maximum likelihood estimation-A commonly used technique to invert winds from scatterometer observations. An improved inversion scheme, which filters the anomalously low RSCAT backscatter measurements, is proposed in Section III. In Section IV, the new inversion scheme is validated using collocated ECMWF and ASCAT wind data. Finally, the conclusions are presented in Section V.

\section{RSCAT WIND INVERSION}

The JPL L2A backscatter data in Hierarchical Data Format needs to be converted into Binary Universal Form for the Representation (BUFR) format before the wind inversion process using PenWP. Within a certain WVC, all the $\sigma^{\circ}$ measurements of the egg-shaped footprints, hereafter named as eggs, are aggregated into four beams during the conversion, i.e., inner (HHpolarized) fore and aft beams, and outer (VV-polarized) fore and aft beams. Here, VV and $\mathrm{HH}$ denote the polarization combination of the transmitted and the received radar signals, i.e., $\mathrm{V}$ for vertical polarization and $\mathrm{H}$ for horizontal polarization. In that case only the VV-polarized measurements are available over the outer swath, the VV fore and aft eggs are redistributed over four azimuthal beams [3], such that each beam may only consist of one egg measurement.

The sea-surface winds are retrieved by minimizing the MLE cost function [6], [7] below

$$
\text { MLE }=\sum_{i}^{N} \frac{\left(\sigma_{m i}^{0}-\sigma_{s i}^{0}\right)^{2}}{\left(K_{p i} \cdot \sigma_{t i}^{0}\right)^{2}}
$$

where $N=4$ is the number of measurements at different azimuthal angles (or beams), $\sigma_{m i}^{0}$ is the backscatter measured by the $i$ th beam, $\sigma_{s i}^{0}$ is the backscatter simulated through the NSCAT-4 GMF [8], [9], $\sigma_{t i}^{0}$ is the "true" backscatter value, and $K_{p i}$ is the normalized standard deviation of the backscatter measurements [10]. Here, the NSCAT GMF is used to process RSCAT data rather than the QuikSCAT GMF (QSCAT-1), because the former is more capable than the latter in removing unrealistic ambiguous wind solutions, by providing a less ambiguous wind product without decreasing the quality of the wind retrieval [9], [11]. The denominator of (1) represents the true error variance. In practice, $\sigma_{t i}^{0}$ is unknown, and it is taken from either $\sigma_{m i}^{0}$ or $\sigma_{s i}^{0}$. The latter leads to an estimated error variance being proportional to $\sigma_{s i}^{0}$, which results in an adaptive noise term during the MLE cost function minimization (inversion), and in turn leads to a positive bias in the retrieved wind speed, notably at low winds [12], [13]. Therefore, PenWP adopts $\sigma_{m i}^{0}$ in the denominator of (1).

The inversion residual or MLE value is used for both QC and ambiguity removal (AR) purposes. On the one hand, a large MLE value indicates the backscatter measurements lie far away from the GMF, and are likely to be contaminated by geophysical effects other than a mean-area wind such as rain, sea ice, or confused sea state. Consequently, an MLE threshold is set to QC poor-quality wind retrievals [5]. On the other hand, the MLE value is related (through an exponential function) to the probability of that particular wind solution being the true wind [14]. In general, the MLE minimization leads to up to four welldefined minima, i.e., wind solutions or ambiguities. However, in case of poor azimuth diversity or noisy scatterometer systems such as RSCAT, the inversion leads to broad (thus rather undefined) minima, i.e., with a broad range of almost equally likely wind solutions. In such cases, considering up to four solutions (minima) in the AR step leads to large wind retrieval errors [11], [15]. An effective way to use the full information content from inversion (up to 144 wind solutions) during AR is to use the Multiple Solution Scheme (MSS) [15]. This scheme uses the multiple solution inversion output in combination with a two-dimensional variational analysis AR (2DVAR) scheme, which is not only capable of explicitly using probability for the multiple solutions but also ensures spatial consistency and meteorological balance of the retrieved winds [16].

\section{PRoblem AND IMPRovement}

Fig. 2(a) illustrates one case with spuriously low retrieved speed on January 20, 2015, at UTC 08:07. The solid curve shows the wind speed values that minimize (1) at different wind direction intervals and the dashed curve is the corresponding MLE value versus wind direction (see the right $y$-axis). The two square markers indicate the local minima of the MLE cost function. The diamond marker indicates the wind solution selected by PenWP using MSS and 2DVAR. As already mentioned, the selected wind does not have to coincide with an MLE cost function minimum using MSS. Looking at the input set of backscatter values $[-42.65,-14.01,-17.88,-18.49] \mathrm{dB}$ (from left to right side, each value represents the measu rement by $\mathrm{HH}$ fore 


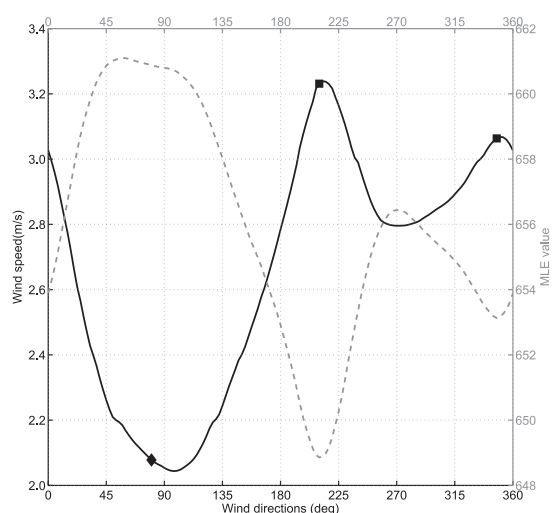

(a)

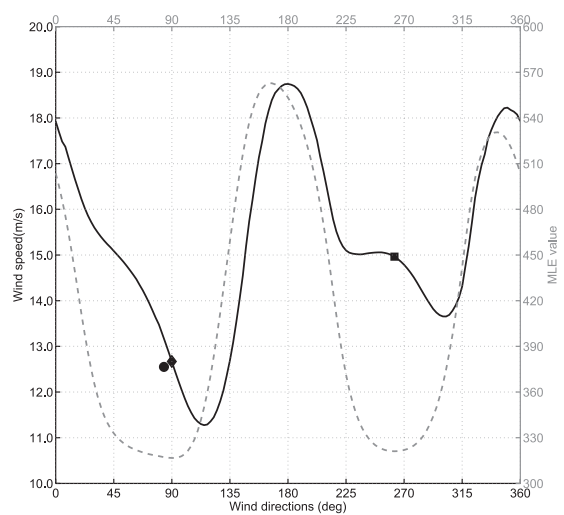

(b)

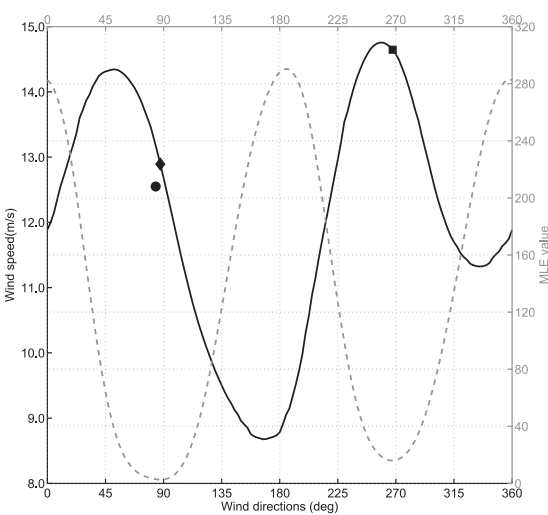

(c)

Fig. 2. Wind speed value that minimizes the MLE cost function at different wind direction (solid curve), and its corresponding MLE value (dashed curve) for (a) nominal inversion (i.e., with all input $\sigma^{\circ}$ measurements); (b) alternative inversion using $\sigma_{s i}^{0}$ in the denominator (also with all input $\sigma^{\circ}$ measurements); (c) improved inversion with filtering anomalously low $\sigma^{\circ}$ values. Note that the square markers indicate the local minima, the diamond marker indicates both the local minimum and the selected solution, and the circle marker shows the reference ASCAT wind vector.

beam, VV fore beam, HH after beam, and VV after beam), it is clear that the $\mathrm{HH}$ fore-beam $\sigma^{\circ}$ value is much lower than the other backscatter values. Since $K_{p}$ values of the four beams are almost comparable (not shown), it is clear that when using the PenWP MLE cost function (i.e., that with $\sigma_{m i}^{0}$ in the denominator), the $\mathrm{HH}$ fore beam term with a very low value in the denominator will dominate, thus leading the minimization toward very low backscatter values and, in turn, to very low retrieved wind speeds.

There are two alternative ways to improve the wind inversion in Fig. 2(a). First, one can use $\sigma_{s i}^{0}$ instead of $\sigma_{m i}^{0}$ as $\sigma_{t i}^{0}$ in the denominator of (1). In this case, the error variance is proportional to the simulated $\sigma_{s i}^{0}$ such that the inversion tends to produce wind solutions with increased speed and decreased MLE values than the PenWP nominal retrieval procedure, as shown in Fig. 2(b). For this anomalously low backscatter case, the retrieved wind vector is closer to the ASCAT wind reference (see the cycle marker) than for the PenWP nominal inversion procedure. However, the MLE value is still much higher than the PenWP QC threshold; hence, this WVC is flagged as poorquality wind and will not be used in the 2DVAR step. Assigning a "wrong" QC flag to certain WVC impacts not only the assessment of a particular WVC wind quality, but also the wind selection for the neighboring WVCs. Moreover, as already mentioned, this alternative MLE formulation generally leads to positive wind speed biases at low winds for nominal conditions, i.e., without any anomalously low backscatter value in the measurement set. Therefore, it is desirable to find a better alternative to address these anomalous cases.

The second alternative is to exclude the anomalously low $\sigma^{\circ}$ value, and simply modify the inversion scheme to run with less than four beams in PenWP. Fig. 2(c) shows the wind inversion without the anomalously low $\sigma^{\circ}$ of $\mathrm{HH}$ fore beam. Two well-defined local minima are present in the cost function, one of them (that selected by 2DVAR) being also close to the ASCAT reference. This therefore seems to be an optimal improvement to the inversion for a measurement set with anomalously low $\sigma^{\circ}$ value(s). The critical point then becomes to identify the

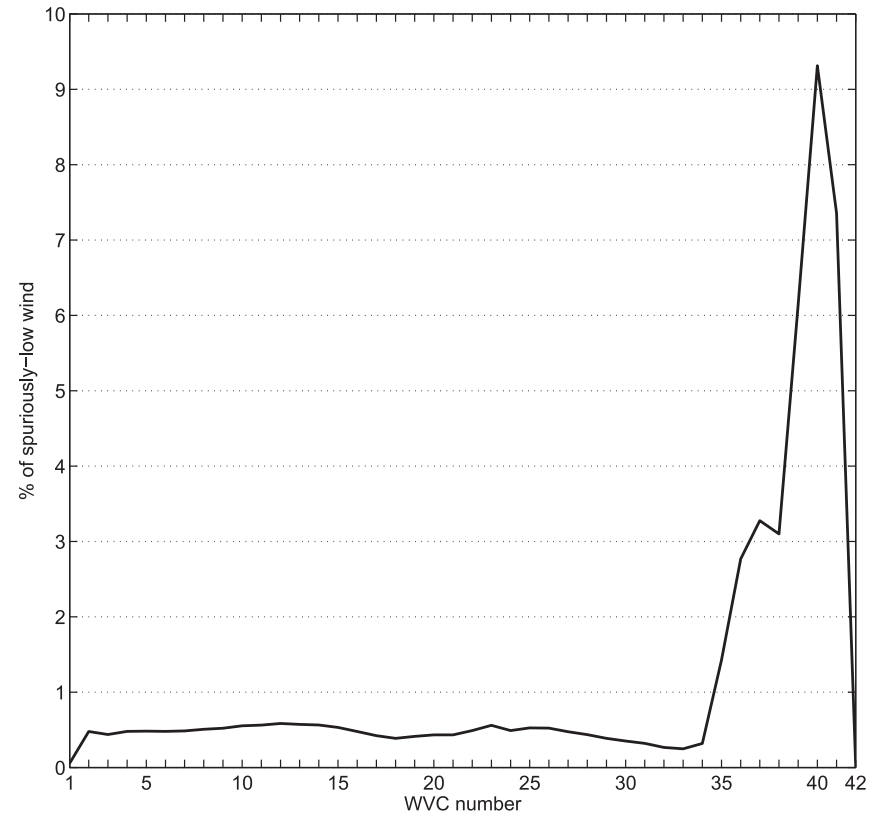

Fig. 3. Percentage of spuriously low winds within the RSCAT QC-rejected data as a function of WVC number. The spurious case is arbitrarily defined as RSCAT $w<4 \mathrm{~m} / \mathrm{s}$ and ECMWF $w>10 \mathrm{~m} / \mathrm{s}$.

anomalously low $\sigma^{\circ}$ value(s) within the input backscatter set and exclude them before inversion.

The characteristics of the spuriously low winds are then studied in order to seek an effective method for identifying them, and in turn, improving the RSCAT wind inversion. Fig. 3 shows the spatial distribution of those poorly inverted low winds arbitrarily defined as $w_{\mathrm{RSCAT}}<4 \mathrm{~m} / \mathrm{s}$ and $w_{\mathrm{ECMWF}}>10 \mathrm{~m} / \mathrm{s}$ (see the bottom contour in Fig. 1), which is not exact but represent most of the spuriously low winds. They are usually in the right-outer swath (i.e., in one of the VV-pol beams). This may be due to partial blocking of one of the four beams by some physical structures (such as solar panels), such that the effective number of eggs over the right-outer swath is usually lower than that over other swath regions unaffected by such 


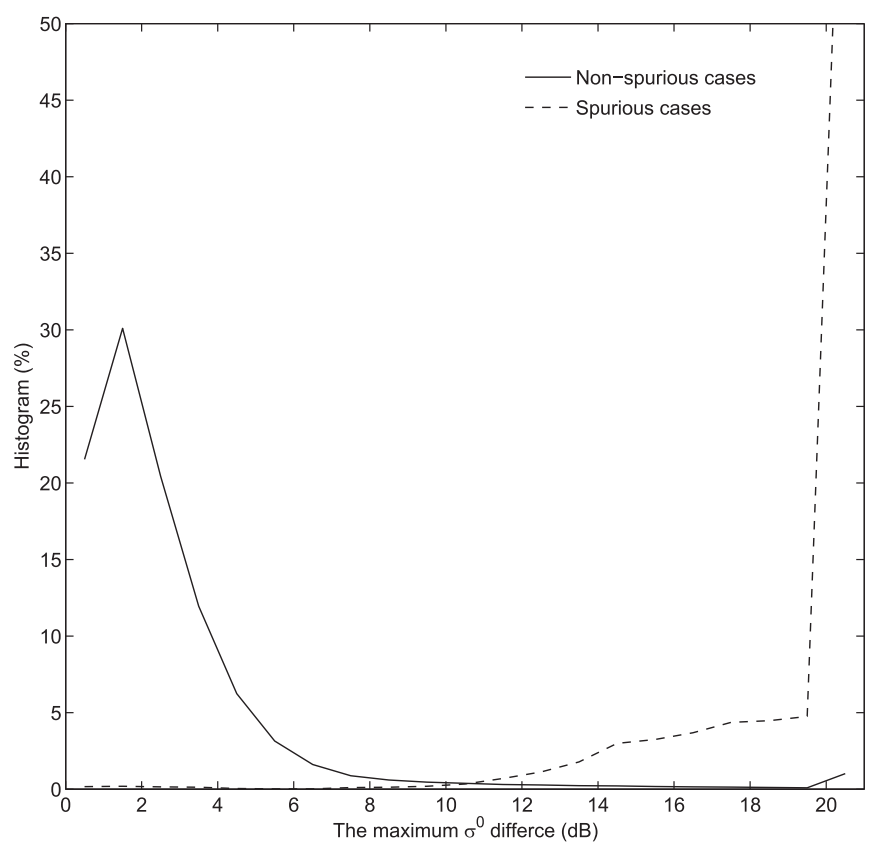

Fig. 4. Normalized histogram of maximum $\sigma^{\circ}$ difference within the input backscatter set for nonspurious (solid curve) and spurious cases (dashed).

blocking effect. Note that for RSCAT a number of eggs in the range of [2], [5] is usually integrated for each beam. When the total amount of eggs on a WVC is lower than for the nominal case, there may be one or two beams which only produce one egg for that particular WVC. Such one-egg beam(s) is (are) thus generally of higher uncertainty than the other beams. Deficient eggs also occur in the southernmost WVCs (not shown) or the start of a RSCAT data gap (see Section IV-B), where the actual forward observations are usually missing, therefore producing spuriously low winds that span across the entire swath with a circular pattern. Another possibility for the anomalous cases is the platform attitude: the onboard processing of RSCAT accounts for ISS nominal attitude variations; however, such variations sometimes exceed those accounted by the nominal processing, leading to very low backscatter values in one of the RSCAT four beams, which in turn lead to spuriously low retrieved winds.

Fig. 4 presents the normalized histogram of maximum $\sigma^{\circ}$ difference within the input VV-polarized backscatters. The curve for HH-polarized measurements is similar, hence it is not shown. The anomalous $\sigma^{\circ}$ value is usually $10 \mathrm{~dB}$ lower than the maximum backscatter. In practice, we propose to reject a low backscatter according to the three criteria below

$$
\left\{\begin{array}{l}
\max \left\{\sigma_{m i}^{0}\right\}-\sigma_{m}^{0}>10 \mathrm{~dB} \\
\max \left\{\sigma_{m i}^{0}\right\}>-30 \mathrm{~dB} \\
\frac{\left(\sigma_{m}^{0}-\sigma_{s}^{0}\right)^{2}}{\left(K_{p} \cdot \sigma_{m}^{0}\right)^{2}}>1000
\end{array} .\right.
$$

The first criterion is set according to the above discussion. The second is set to avoid low wind conditions (below $4 \mathrm{~m} / \mathrm{s}$ ), since we are not able to distinguish between the anomalously low backscatters and the normal low echoes from calm sea surface.

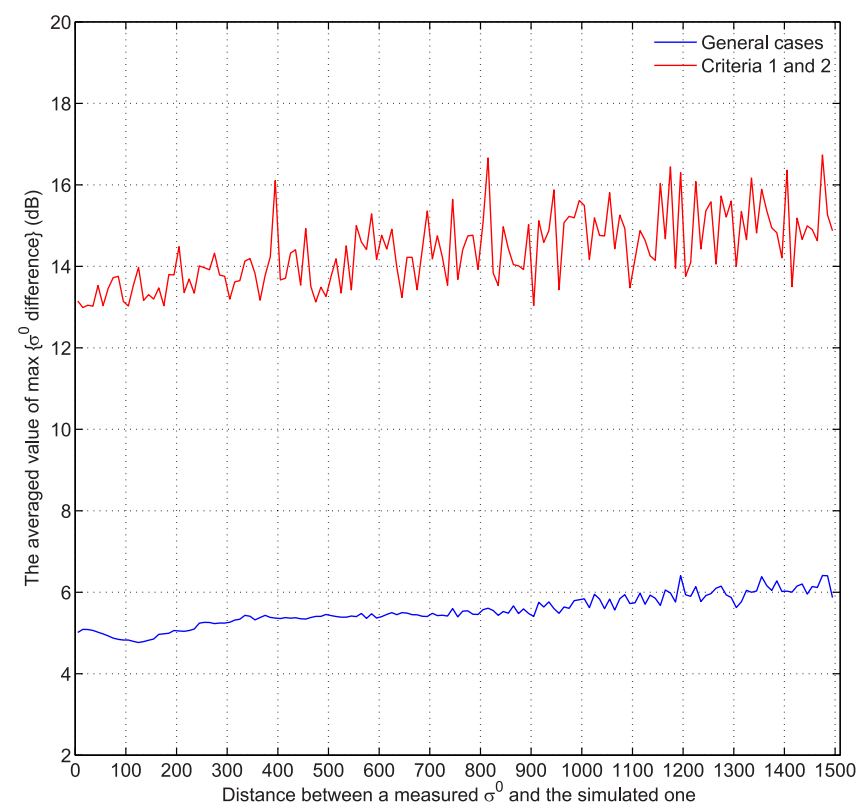

(a)

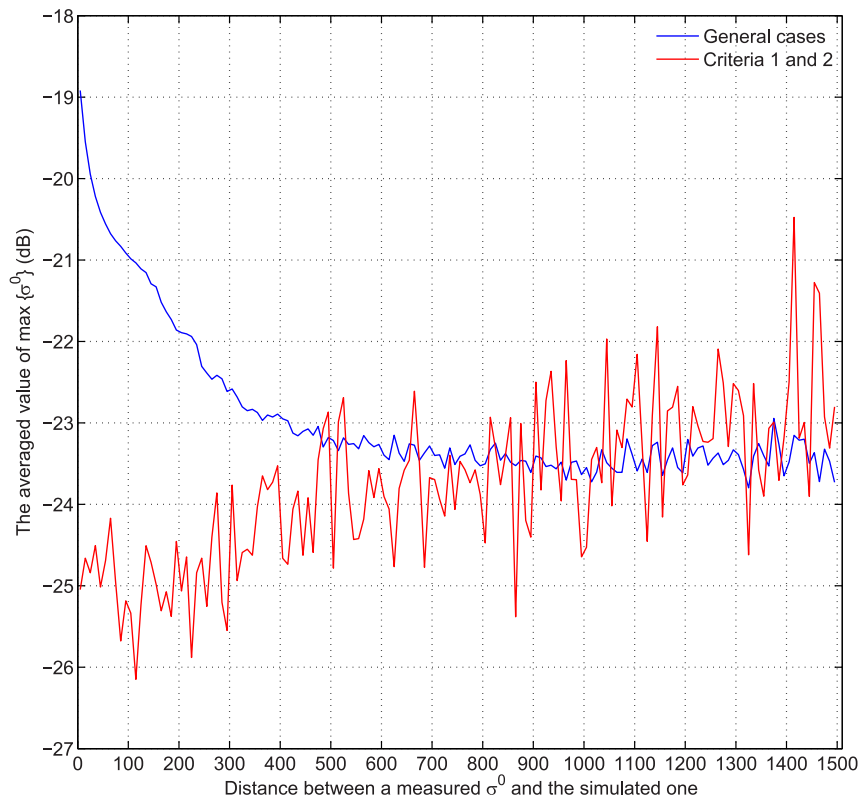

(b)

Fig. 5. Average value of the maximum $\sigma^{\circ}$ difference [see first criterion in (2)] (a) and the maximum $\sigma^{\circ}$ value [see second criterion in (2)] (b) as a function of the distance between a measured $\sigma^{\circ}$ and the simulated $\sigma^{\circ}$ [see third criterion in (2)]. The binning is set to 10 .

The third is set to account for interbeam inconsistencies before inversion, where $\sigma_{s}^{0}$ is simulated from the collocated ECMWF background wind. Normally, the left side of the third criterion is less than 200, while such value could be much larger than 200 when an anomalously low $\sigma^{\circ}$ value presents (not shown). In fact, the first and second criteria already lead to $\sigma^{\circ}$ values which are generally very far away from the simulated $\sigma^{\circ}$ values and thus inconsistent with the other backscatter measurements. Fig. 5(a) shows the averaged value of the maximum $\sigma^{\circ}$ difference as a function of the distance between a measured $\sigma^{\circ}$ value 


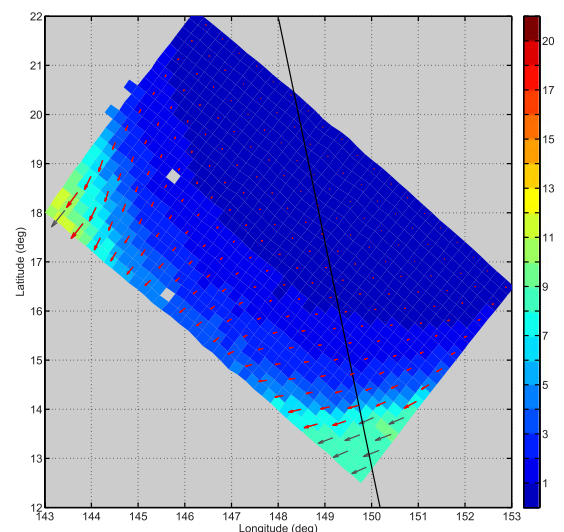

(a)

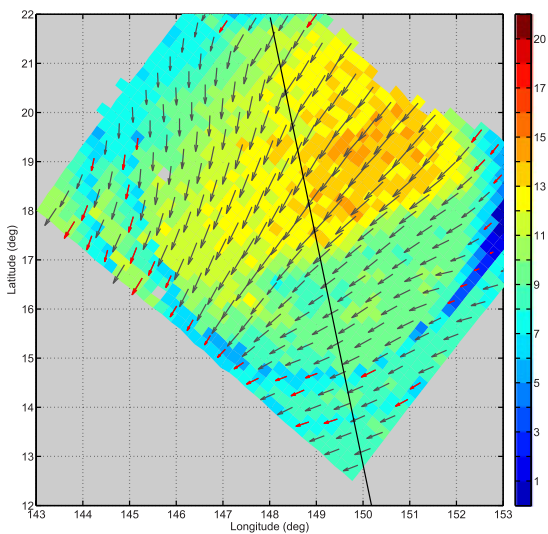

(b)

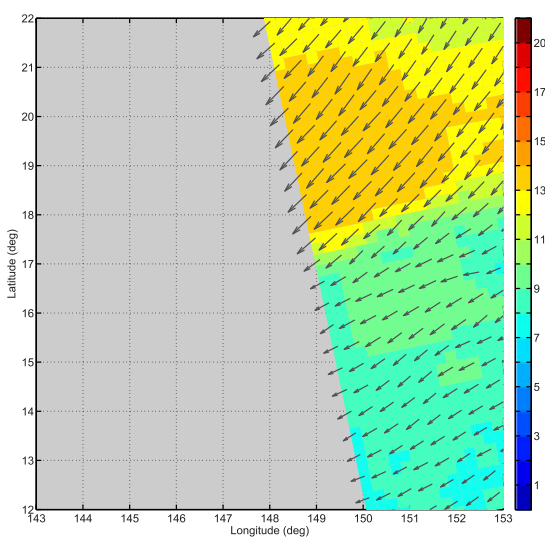

(c)

Fig. 6. (a) RSCAT wind field retrieved with the nominal PenWP inversion. The acquisition date and time are January 25th 2015, at 11:52 UTC; (b) RSCAT wind field retrieved with the improved inversion; (c) Collocated ASCAT wind field was acquired on the same day, at 11:36 UTC. The gray arrows indicate QC-accepted winds, and the black arrows indicate QC-rejected winds. The colorbar corresponds to the retrieved wind speed [m/s] for each scene.

and the simulated $\sigma^{\circ}$ value. Here, the binning is set to 10 (note that the distance as defined in the left side of third criterion is dimensionless), while the bins above 1500 are not shown. It verifies that the spurious cases (dashed curve) are clearly discriminated from the general dataset (solid curve). Fig. 5(b) illustrates the averaged value of the maximum $\sigma^{\circ}$ as a function of the above-mentioned distance. It shows that the spurious cases with relatively small distances (see the dashed curve for values below 500) are actually close to the threshold of the second criterion, i.e., they correspond to relatively low wind speeds. Since it is difficult to distinguish between the anomalously low backscatters and the nominal low echoes at low wind conditions, we propose a third criterion to consolidate the second [see (2)]. Although the threshold for the third criterion is set at 1000, one may argue that between 500 and 1000 the wind speeds associated with the dashed curve dataset are not much lower than those associated with the solid curve dataset (both the dashed and solid curves have similar values in this interval). However, note that setting the threshold at 500 or 1000 does not make much difference since there are very few WVCs within that range (less than $4 \%$ over the cases determined by Criteria 1 and 2). Note that there may be other more robust way to identify the anomalous $\sigma^{\circ}$ value, such as using the retrieved wind speed instead of ECMWF background wind. However, this needs to do twice the wind inversion for the spuriously low wind cases, and it is not applicable for the near real time process.

\section{RESULTS}

\section{A. Data}

The dataset in this section consists of one year (2015) of RSCAT 25-km L2 data collocated with ECMWF and ASCAT $25-\mathrm{km}$ gridded winds. The ASCAT data are used as reference, because the inclined ISS orbit allows collecting a large amount of ASCAT collocations within a short period of time. Meanwhile, it has been proven that subcell wind variability rather than rain is the dominant source of wind quality degradation in $\mathrm{C}$ band ASCAT [17]. At the scatterometer-resolved spatial scale,
ASCAT provides better quality winds than either ECMWF or buoy measurements even for high subcell wind variability [17]. To be rigorous, only the ASCAT QC-accepted data (99.1\%) are used in the following validation.

Both RSCAT and ASCAT data are processed at KNMI and disseminated in BUFR format in the context of EUMETSAT OSI SAF. The ECMWF background winds are already included in RSCAT L2 BUFR files, which have been acquired by interpolating three ECMWF forecasts selected from +3 to $+18 \mathrm{~h}$ in 3-h steps. The collocation criteria for ASCAT winds are less than $30 \mathrm{~min}$ and $25 \mathrm{~km}$ distance from the RSCAT acquisition, although in practice more than $90 \%$ of collocations are within $15 \mathrm{~km}$. In case more than one ASCAT WVC meets the collocation criteria for a particular RSCAT WVC, only the closest in space is used.

\section{B. Typical Example}

Fig. 6(a) presents the RSCAT wind field observed on January 25, 2015, around 11:52 UTC, as retrieved with the PenWP nominal settings. The gray and black arrows indicate QC accepted and rejected data, respectively. Almost all the low winds are rejected. Fig. 6(b) shows the RSCAT wind field retrieved by the improved scheme with prefiltering the input backscatters using (2), in which most of the spuriously low winds have been corrected except a few WVCs in the right-outer swath. For the sake of comparison, the solid lines in (a) and (b) correspond to the swath edge of the collocated ASCAT overpass. The new RSCAT winds are in good agreement with the reference ASCAT winds shown in Fig. 6(c), and they are generally accepted by the nominal QC module. Moreover, the improved inversion produces a slightly larger coverage than the default one. That is because in case there are one or more extremely low $\sigma^{\circ}$ values (e.g., $<-70 \mathrm{~dB}$ ) within the input backscatter set, the nominal process always reaches the GMF lower boundary $(0.2 \mathrm{~m} / \mathrm{s})$ when minimizing the MLE cost function at different wind direction. Therefore, no wind selection is performed for such particular condition, and in turn, the swath coverage tends to be reduced. Wind inversion using less than four good-quality 
TABLE I

DiRECT INFLUENCE OF THE IMPROVED INVERSION ON THE QC-REJECTED WVCS (LEFT TwO COLUMNS) AND THE WIND-UNAVAILABLE WVCS (RIGHT TWO Columns) Associated With the Nominal Process. In the First Column, THE Vector RoOt-Mean-SQuare (VRMS) SCORES OF the NOMinal INVERSION ARE SHOWN IN PARENTHESIS

\begin{tabular}{|c|c|c||c|c|}
\hline After improvement & $\begin{array}{c}\text { VRMS }[\mathrm{m} / \mathrm{s}] \text { w.r.t. } \\
\text { ECMWF }\end{array}$ & $\begin{array}{c}\text { Ratio } \\
\frac{\text { The improved QC-rejected data }}{\text { The nominal QC-rejected data }} \\
(\%)\end{array}$ & $\begin{array}{c}\text { VRMS [m/s] w.r.t. } \\
\text { ECMWF }\end{array}$ & $\begin{array}{c}\text { Ratio }= \\
\frac{\text { The improved wind-unavailabledata }}{\text { The nominal QC-rejected data }} \\
(\%)\end{array}$ \\
\hline QC-accepted & $2.34(7.16)$ & 4.67 & 3.42 & 0.11 \\
\hline QC-rejected & $3.86(7.91)$ & 0.99 & 6.97 & 0.05 \\
\hline
\end{tabular}

In the second and fourth columns, the ratios over the nominal QC-rejected data are shown.

TABLE II

INDIRECT INFLUENCE OF THE IMPROVED WIND INVERSION ON THE NEIGHBORING WVCS (THROUGH 2DVAR)

\begin{tabular}{|c|c||c|}
\hline & VRMS [m/s] w.r.t. ECMWF & Ratio $=\frac{\text { The affected neighbouring data }}{\text { The QC-accepted or rejected data }}(\%)$ \\
\hline QC-accepted & $1.95(1.94)$ & 1.89 \\
\hline QC-rejected & $4.04(4.02)$ & 3.31 \\
\hline
\end{tabular}

In the first column, the VRMS differences of the nominal inversion are shown in parenthesis. The second column shows the percent of the affected neighboring data over each row category.

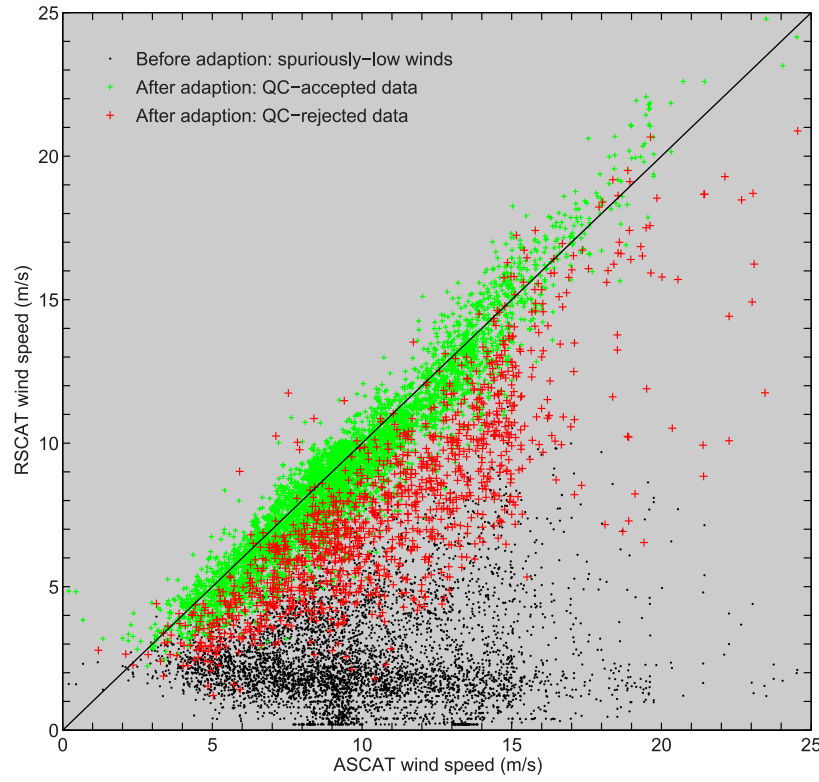

Fig. 7. Scatter plot of RSCAT versus ASCAT winds for the cases with anomalously low backscatter(s), as filtered by (2). The black dots show the results from the nominal PenWP RSCAT inversion scheme, while the gray and white crosses indicate the RSCAT QC-accepted and QC-rejected winds, respectively, using the improved inversion scheme.

$\sigma^{\circ}$ values will also produce four well-defined local minima. Although such inversion is not optimal for backscatter duplets, the PenWP 2DVAR is able to select the optimal solution when the ECMWF background is well specified [11].

\section{General Validation}

The improved inversion is first evaluated using 1 month of RSCAT data (January 2015) collocated with ECMWF winds. The results show that the prefiltering scheme (2) is rarely

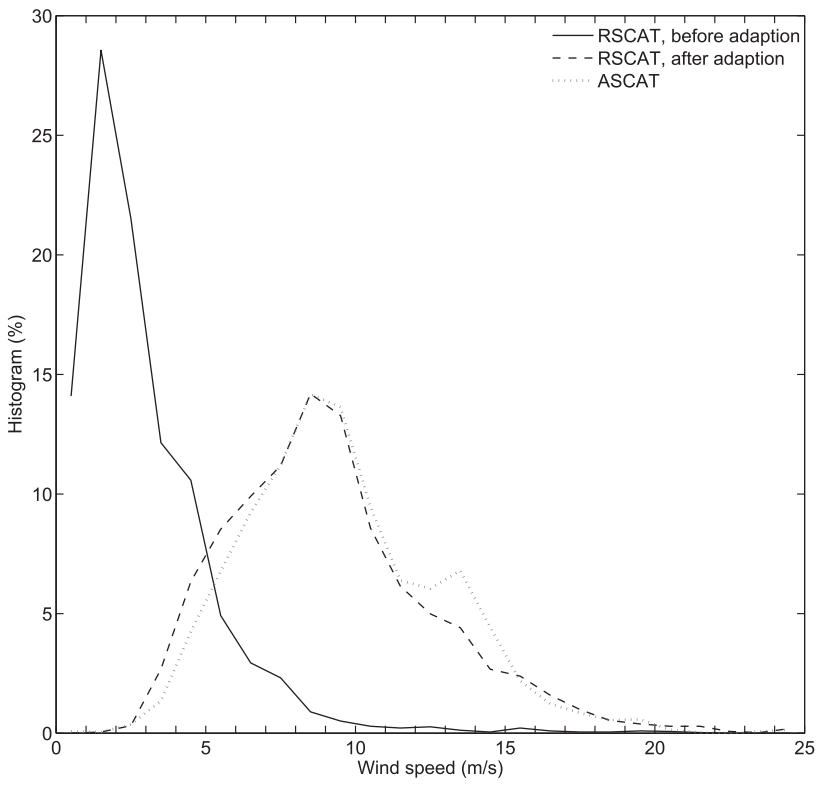

Fig. 8. Wind speed histograms of the spurious cases rejected by the nominal PenWP inversion (solid curve), but accepted by the improved inversion (dashed curve). The dotted curve shows the speed histogram of collocated ASCAT winds (bins of $1 \mathrm{~m} / \mathrm{s}$ ).

activated for nominal QC-accepted data (less than 1000\%). Nonetheless, corrections carried out in the QC-rejected data are not negligible $(0.3 \%$ in total, and $5.7 \%$ in the rejected category). As discussed previously, producing more QC-accepted data is not only desirable in terms of data coverage, it also affects the 2DVAR for the neighboring WVCs. Consequently, the impact of the proposed method on RSCAT wind quality is separately assessed for the directly affected WVCs in Table I and for the indirectly affected neighboring WVCs (i.e., those WVCs in which the selected wind ambiguity using the nominal scheme differs from that using the improved scheme) in Table II. 
TABLE III

WIND SPEED BIAS [M/s] AND VRMS [M/s] BETWEEN RSCAT AND ECMWF WINDS FOR DIFFERENT CATEGORIES OF THE COLLOCATED RSCAT, ASCAT, AND ECMWF DATASET

\begin{tabular}{|c|c|c|c|c|c|c|}
\hline & \multicolumn{4}{|c|}{ Directly affected WVCs } & \multirow{2}{*}{\multicolumn{2}{|c|}{ Indirectly affected neighboring WVCs }} \\
\hline Afte & \multicolumn{2}{|c|}{ Winds available in nominal inversion } & \multicolumn{2}{|c|}{$\begin{array}{l}\text { Winds unavailable in } \\
\text { nominal inversion }\end{array}$} & & \\
\hline & Bias & VRMS & Bias & VRMS & Bias & VRMS \\
\hline QC-accepted & $-0.44(-6.66)$ & $1.94(7.63)$ & -0.83 & 2.98 & $0.16(0.16)$ & $1.91(1.88)$ \\
\hline QC-rejected & $-3.01(-8.11)$ & $4.19(9.19)$ & -7.01 & 7.53 & $1.26(1.14)$ & $4.07(3.96)$ \\
\hline
\end{tabular}

Effects of the proposed inversion are evaluated for the directly and indirectly affected WVCs, respectively. In parenthesis, the same scores but for the nominal PenWP inversion scheme are shown.

TABLE IV

SAME AS TABLE III, BUt USING ASCAT WINDS INSTEAD OF ECMWF BACKGROUND AS THE REFERENCE

\begin{tabular}{|c|c|c||c|c||c|c|}
\hline \multirow{2}{*}{$\begin{array}{l}\text { After } \\
\text { improvement }\end{array}$} & \multicolumn{5}{|c||}{ Directly affected WVCs } & \multicolumn{2}{c|}{ Indirectly affected neighboring WVCs } \\
\cline { 2 - 6 } & \multicolumn{2}{|c|}{ Winds available in nominal inversion } & $\begin{array}{c}\text { Winds unavailable in } \\
\text { nominal inversion }\end{array}$ & \\
\cline { 2 - 6 } & Bias & VRMS & Bias & VRMS & Bias & VRMS \\
\hline QC-accepted & $-0.41(-6.62)$ & $1.86(7.64)$ & -1.11 & 2.53 & $0.06(0.06)$ & $1.73(1.74)$ \\
\hline QC-rejected & $-3.04(-8.15)$ & $4.17(9.17)$ & -7.42 & 7.84 & $0.45(0.34)$ & $4.63(4.53)$ \\
\hline
\end{tabular}

Regarding the direct influence, the improved inversion accepts over $80 \%$ of the WVCs that meet the criteria in (2). The remaining $20 \%$ has relatively high MLE values and is therefore rejected. Moreover, the improved inversion leads to RSCAT winds more in agreement with ECMWF winds than the nominal PenWP inversion (see the left two columns of Table I). Occasionally, the proposed inversion produces a few additional wind data which are not retrieved by the nominal process, as explained in Section IV-B, and the statistics are shown in the right two columns of Table I. Concerning the indirect influence, there are $1.89 \%$ of QC-accepted data and $3.31 \%$ of QC-rejected data affected by the spatial filter 2DVAR. The affected WVCs have a slightly higher VRMS score than the nominal WVCs. This is because the more data used in 2DVAR (with more poorquality winds), the larger discrepancy between the selected and the ECMWF winds, and vice versa [16]. The improved inversion reserves more WVCs than the nominal one according to the same wind QC; these additional QC-accepted WVCs (see the first row of Table I), which are of slightly higher VRMS scores as compared to the general score (i.e., $1.80 \mathrm{~m} / \mathrm{s}$ ), are then used by 2DVAR, and in turn affect the wind selection of the neighboring WVCs. However, this does not necessarily mean that the affected neighboring RSCAT (QC-accepted) WVCs are of lower quality when using the new improved inversion, since ECMWF winds are not the best reference at scatterometer scale. A more reliable reference is the ASCAT wind product, which is used to further evaluate the above-mentioned wind quality.

Fig. 7 illustrates how the spuriously low winds are corrected by the improved scheme, using ASCAT winds as reference. Since the proposed inversion does not affect the selected wind direction remarkably due to the MSS effect (not shown), we mainly present the changes in the wind speed component. The black dots indicate the spuriously low winds derived from the nominal PenWP inversion. The gray and white crosses depict the QC-accepted and QC-rejected data after applying the improved inversion, respectively. It is clear that the PenWP QC well separates the good-quality WVCs (close to the diagonal) from the poor-quality WVCs (away from the diagonal). In Fig. 8, the wind speed histograms corresponding to the gray crosses in Fig. 7 are shown for both the nominal PenWP inversion (solid line) and the improved inversion (dashed line). It is also clear that the latter is much closer to the ASCAT wind speed distribution than the former.

Tables III and IV summarize the impact of the proposed inversion using RSCAT-ASCAT-ECMWF collocations. The ratio of data in each category is different with those in Tables I and II, e.g., the percentage of direct corrections over the total amount of collocations is about $0.23 \%$, and the ratio of indirectly affected neighboring WVCs is $7.5 \%$ for QC-accepted data and $9.7 \%$ for QC-rejected data. This is probably due to the following reasons: 1) the geographical distribution of the RSCAT-ASCAT dataset is different from the general dataset used in Tables I and II. The former has a higher percentage of data than the latter at high latitudes (beyond $45^{\circ} \mathrm{N} / \mathrm{S}$, not shown); 2) due to the tropical rain, the QC rejection ratio is higher in the Tropics than in the other regions [5]; and 3) the spuriously low winds generally occur at the start of a RSCAT data gap, particularly at South Hemisphere high latitudes (not shown), since each orbital file starts at the southernmost location. Nonetheless, the VRMS scores in Table III behave the same trends as Tables I and II, notably for the indirectly affected WVCs. The evaluation against ASCAT winds in Table IV, however, shows that wind quality of the affected neighboring WVCs (QC-accepted) actually does not degrades; even there is a slightly positive influence. Moreover, it shows RSCAT QC-accepted winds are generally closer to ASCAT winds than the ECMWF background, since the two 
TABLE V

DiRECTLY AFFECTED WVCS By THE IMPROVED INVERSION ARE FURTHER EXAMINED IN TwO DifFERENT CATEGORIES: THE FIRST ROW SHOWS THE STATISTICS for Those WVCs Having Only One Anomalously low $\sigma^{\circ}$ Value; the Second Row Shows the Statistics for Those WVCs Having Two ANOMALOUSLY LOW $\sigma$ VALUES (IN PARENTHESIS, THE SAME SCORES OF THE NOMINAL INVERSION ARE SHOWN)

\begin{tabular}{|c|c|c|c|}
\hline \multicolumn{2}{|c|}{ Number of abnormal $\sigma^{0}$ valtes } & VRMS w.r.t. ASCAT $[\mathrm{m} / \mathrm{s}]$ & Ratio (\%) over the adapted data \\
\hline \multirow{2}{*}{1} & QC-accepted & $1.84(6.68)$ & 45.1 \\
\cline { 2 - 4 } & QC-rejected & $4.22(9.28)$ & 23.7 \\
\hline \hline \multirow{2}{*}{2} & QC-accepted & $1.93(9.24)$ & 30.9 \\
\cline { 2 - 4 } & QC-rejected & $3.86(9.92)$ & 0.3 \\
\hline
\end{tabular}

Second column presents the data ratios over the category of directly affected WVCs.

scatterometers resolve similar spatial scales that are both smaller than ECMWF. In summary, the proposed inversion improves the wind quality for the spuriously low wind cases remarkably.

Finally, Table V further examines the directly affected WVCs according to the number of anomalous backscatters filtered by the criteria (2). In case of nominal wind version without filtering any backscatter, the QC rejection ratio is about $5 \%$, and the VRMS difference between RSCAT and ASCAT winds is $1.59 \mathrm{~m} / \mathrm{s}$ for the QC-accepted data, and $4.54 \mathrm{~m} / \mathrm{s}$ for the QCrejected data (not shown). In case of filtering one anomalous backscatter, the QC rejection ratio is about $24 \%$, and the VRMS value of QC-accepted data is lower than the nominal inversion. Furthermore, almost all the WVCs are accepted by PenWP QC if two anomalously low backscatters are filtered in the inversion, and the VRMS value of QC-accepted data is even lower than the above two types of inversion. In other words, the VRMS value of QC-accepted (-rejected) data increases (decreases) as the number of input backscatter measurements decreases, i.e., it becomes more challenging to distinguish between good- and poor-quality winds when the inversion problem is ill-posed. This suggests that the wind QC performance degrades as the number of input backscatter measurement decreases.

\section{CONCLUSION}

This paper reviews the wind retrieval procedure for Ku-band pencil-beam scatterometers, and addresses an unprecedented problem manifested in the RSCAT L2 data processed by PenWP. Due to the presence of anomalously low $\sigma^{\circ}$ value(s) within the input backscatter set, the nominal PenWP inversion scheme produces extremely low winds of poor quality, which are subsequently rejected by the PenWP QC. The characteristics of these spurious cases are studied. It is revealed that the spuriously low winds usually appear when a certain beam of the input backscatter set only consists of one egg-shaped measurement. That is the case for the right-outer swath, probably due to the blocking of one of the four beams by some ISS physical structures; and for the southernmost location or the start of RSCAT data gap, where the forward observations are usually missing. An improved inversion is proposed, which automatically identifies and rejects the anomalously low $\sigma^{\circ}$ before minimizing the cost function. On the one hand, the proposed inversion remarkably improves the retrieved wind quality of those directly affected WVCs. On the other hand, the proposed inversion shows a slightly positive impact on the wind quality for the neighboring WVCs after 2DVAR, i.e., the neighboring winds become slightly closer to ASCAT winds. Note though that the neighboring winds are more discrepant to ECMWF winds. However, as discussed in Section IV-C, ASCAT winds are a better reference than ECMWF at scatterometer scales.

Although the proposed method only corrects a small percent of RSCAT data (about $0.3 \%$ in total), its impact is significant. First, the spuriously low winds are usually patchy, thus affecting wide areas on the surface. Although the PenWP is effective in rejecting such cases, such data gaps can have a potential negative impact in applications such as nowcasting. Second, such spurious winds are caused by the anomalies associated with the instrument, rather than the nonwind geophysical conditions not modeled in GMF. Removing such wind outliers is the prerequisite to assess the data quality in a meaningful way, and to develop an effective QC method. However, this paper does not revisit the wind QC procedure for the improved inversion scheme, even though the validations in Section IV-C imply that the nominal QC approach is imperfect in separating the good quality winds from the poor quality ones for the affected WVCs. A thorough analysis on the Ku-band wind quality (control) will be carried out in the future. Moreover, while the current filter of anomalously low backscatter(s) is performed over the WVC-level $\sigma^{\circ} \mathrm{s}$, a more flexible way to filter the backscatter measurement(s) on egg level could be straightforwardly implemented, provided that egg level data become available.

\section{ACKNOWLEDGMENT}

The authors would like to thank the three anonymous reviewers who helped to improve the paper.

\section{REFERENCES}

[1] C. L. Wu, Y. Liu, K. H. Kellogg, K. S. Pak, and R. L. Glenister, "Design and calibration of the SeaWinds scatterometer," IEEE Trans. Aerosp. Electron. Syst., vol. 39, no. 1, pp. 94-109, Jan. 2003.

[2] J. Vogelzang, A. Verhoef, J. Verspeek, J. de Kloe, and A. Stoffelen, "SDP user manual and reference guide version 2.2," KNMI, De Bilt, The Netherlands, NWP SAF Rep. NWPSAF-KN-UD-002, Sep. 2014. [Online]. Available: http://projects.knmi.nl/publications/index.php. Accessed on: Oct. 9, 2016

[3] A. Verhoef, J. Vogelzang, J. Verspeek, and A. Stoffelen, "PenWP user manual and reference guide," KNMI, De Bilt, The Netherlands, NWP SAF Rep. NWPSAF-KN-UD-009, Nov. 2015. [Online]. Available: http://projects.knmi.nl/publications/index.php. Accessed on: Oct. 9, 2016

[4] A. Verhoef, J. Vogelzang, and A. Stoffelen, "RapidScat wind validation report," KNMI, De Bilt, The Netherlands, OSI SAF Tech. Note SAF/OSI/CDOP2/KNMI/TEC/RP/228, Mar. 2015. [Online]. Available: http://projects.knmi.nl/publications/index.php. Accessed on Oct. 9, 2016. 
[5] M. Portabella and A. Stoffelen, "Rain detection and quality control of SeaWinds," J. Atmos. Ocean. Technol., vol. 18, no. 7, pp. 1171-1183, Jul. 2001.

[6] W. J. Pierson, "Probabilities and statistics for backscatter estimates obtained by a scatterometer," J. Geophys. Res., vol. 94, no. C7, pp. 9743-9759, Jul. 1989.

[7] A. Stoffelen and D. Anderson, "Scatterometer data interpretation: Measurement space and inversion," J. Atmos. Ocean. Technol., vol. 14, no. 6, pp. 1298-1313, Dec. 1997.

[8] F. J. Wentz and D. K. Smith, "A model function for the ocean normalized radar cross section at $14 \mathrm{GHz}$ derived from NSCAT observations," J. Geophys. Res., vol. 104, no. C5, pp. 11499-11514, May 1999.

[9] KNMI Scatterometer Team, "NSCAT-4 geophysical model function," [Online]. Available: http://projects.knmi.nl/scatterometer/nscat_gmf/ Accessed on: Jun. 20, 2016.

[10] M. W. Spencer, C. L. Wu, and D. G. Long, "Improved resolution backscatter measurements with the SeaWinds pencil-beam scatterometer," IEEE Trans. Geosci. Remote Sens., vol. 38, no. 1, pp. 89-104, Jan. 2000.

[11] M. Portabella, "Wind field retrieval from satellite radar systems," $\mathrm{Ph} . \mathrm{D}$ Dissertation, Dept. Astronomy Meteorology, Univ. Barcelona, Barcelona, Spain, 2002.

[12] A. Stoffelen and D. Anderson, "Scatterometer data interpretation: Estimation and validation of the transfer function CMOD4," J. Geophys. Res., vol. 102, no. C3, pp. 5767-5780, Mar. 1997.

[13] M. Portabella and A. Stoffelen, "Characterization of residual information for SeaWinds quality control," IEEE Trans. Geosci. Remote Sens., vol. 40, no. 12, pp. 2747-2759, Dec. 2002.

[14] M. Portabella and A. Stoffelen, "A probabilistic approach for SeaWinds data assimilation," Quart. J. Roy. Meteorological Soc., vol. 130, no. 596, pp. 127-159, Jan. 2004.

[15] B. W. Stiles, B. D. Pollard, and R. S. Dunbar, "Direction interval retrieval with thresholded nudging: A method for improving the accuracy of QuikSCAT winds," IEEE Trans. Geosci. Remote Sens., vol. 40, no. 1, pp. 79-89, Jan. 2002.

[16] J. Vogelzang, A. Stoffelen, A. Verhoef, J. de Vries, and H. Bonekamp, "Validation of two-dimensional variational ambiguity removal on SeaWinds scatterometer data," J. Atmos. Ocean. Technol., vol. 26, no. 3, pp. 1229-1245, Jul. 2009

[17] W. Lin, M. Portabella, A. Stoffelen, J. Vogelzang, and A. Verhoef, "ASCAT wind quality under high sub-cell wind variability conditions," J. Geophys. Res. Oceans, vol. 120, no. 8, pp. 5804-5819, Aug. 2015.

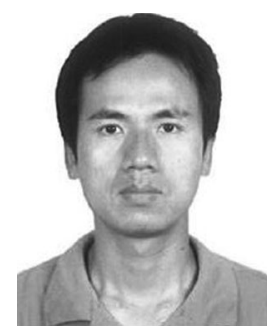

Wenming Lin was born on April 22, 1984, in China. $\mathrm{He}$ received the $\mathrm{B} . \mathrm{Sc}$. degree in engineering from Wuhan University, Wuhan, China, in 2006 and the $\mathrm{Ph} . \mathrm{D}$. degree in engineering from the National Space Science Center, Chinese Academy of Sciences, Beijing, China, in 2011.

He is currently a Postdoctoral Researcher with the Institute of Marine Sciences, Barcelona, Spain, working on the advanced oceanographic data processing methods, remote sensing of ocean surface winds, and data assimilation.

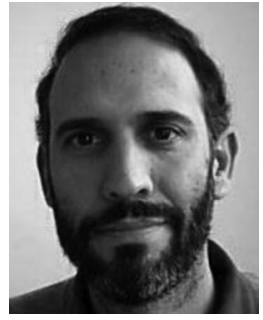

Marcos Portabella was born on October 14, 1970, in Spain. He received the B.Sc. degree in physics from the University of Barcelona, Barcelona, Spain, in 1994, the M.Sc. degree in remote sensing from the Institute of Space Studies of Catalonia, Barcelona, Spain, in 1995, and the Ph.D. degree in physics from the University of Barcelona, Spain, in 2002.

$\mathrm{He}$ is currently with the Institute of Marine Sciences, Barcelona, Spain, working on satellite remote sensing. In particular, he is involved in scatterometry and L-band radiometry.

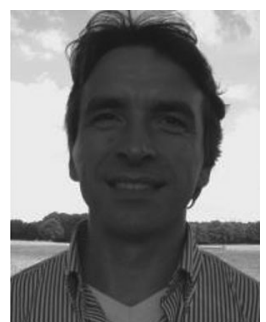

Ad Stoffelen was born on February 25, 1962 in The Netherlands. He received the M.Sc. degree in physics from the Technical University of Eindhoven, Eindhoven, The Netherlands, in 1987 and the Ph.D. degree in meteorology on scatterometry from the University of Utrecht, Utrecht, The Netherlands.

$\mathrm{He}$ is currently working with Royal Netherlands Meteorological Institute and responsible for the ASCAT wind products. His research interests include European Space Agency ADM-Aeolus Doppler Wind Lidar mission.

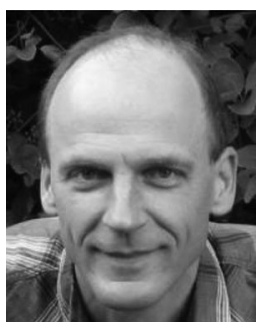

Anton Verhoef was born on Dec 10, 1964, in The Netherlands. He received the M.Sc. degree in physics from Rijksuniversiteit Groningen, Groningen, The Netherlands, in 1989 and the Ph.D. degree in solid state physics from Rijksuniversiteit Groiningen in 1994.

He is currently with Royal Netherlands Meteorological Institute and working on scatterometry processing software development, data validation, quality monitoring, and user services. 